University of Wollongong

Research Online

Australian Institute for Innovative Materials -

Papers

Australian Institute for Innovative Materials

$1-1-2017$

\title{
Human skin interactive self-powered wearable piezoelectric bio-e-skin by electrospun poly-l-lactic acid nanofibers for non-invasive physiological signal monitoring
}

\author{
Ayesha Sultana \\ Jadavpur University \\ Sujoy Ghosh \\ Jadavpur University \\ Vitor Sencadas \\ University of Wollongong, victors@uow.edu.au \\ Tian Zheng \\ University of Wollongong, tz648@uowmail.edu.au \\ Michael J. Higgins \\ University of Wollongong, mhiggins@uow.edu.au
}

See next page for additional authors

Follow this and additional works at: https://ro.uow.edu.au/aiimpapers

Part of the Engineering Commons, and the Physical Sciences and Mathematics Commons

Research Online is the open access institutional repository for the University of Wollongong. For further information contact the UOW Library: research-pubs@uow.edu.au 


\title{
Human skin interactive self-powered wearable piezoelectric bio-e-skin by electrospun poly-l-lactic acid nanofibers for non-invasive physiological signal monitoring
}

\author{
Abstract \\ Flexible and wearable piezoelectric bio e-skin (PBio-e-skin) based on electrospun poly(l-lactic acid) PLLA \\ nanofiber membrane is demonstrated for non-invasive human physiological signal monitoring and \\ detecting dynamic tactile stimuli. The molecular orientations of the $\mathrm{CO}$ dipoles by electrospinning \\ technique result in a longitudinal piezoelectric charge co-efficient (d 33 ) value of $\sim(3 \pm 1) \mathrm{pm} \mathrm{V} \mathrm{-1}$ \\ realized by piezoresponse force microscopy, allowing the PBio-e-skin for pressure sensing applications. \\ The robust mechanical strength (Young's modulus $\sim 50 \mathrm{MPa}$ ) of nanofiber membrane ensures PBio-e- \\ skin's superior operational stability over 375000 cycles. Owing to the superior mechanosensitivity of $\sim 22$ \\ $\mathrm{V} \mathrm{N}-1$, PBio-e-skin has the ability to measure subtle movement of muscle in the internal organs such as \\ esophagus, trachea, motion of joints and arterial pressure by recognition of strains on human skin. This \\ flexible and light weight PBio-e-skin precisely detects vital signs and provides important clinical insights \\ without using any external power source. Eventually, the low cost, environmental friendly PBio-e-skin will \\ have a huge impact in a broad range of applications including self-powered wearable health care systems, \\ human-machine interfacing devices, artificial intelligence and prosthetic skin. \\ Disciplines \\ Engineering | Physical Sciences and Mathematics \\ Publication Details \\ Sultana, A., Ghosh, S. Kumar., Sencadas, V., Zheng, T., Higgins, M. J., Middya, T. Ranjan. \& Mandal, D. \\ (2017). Human skin interactive self-powered wearable piezoelectric bio-e-skin by electrospun poly-l-lactic \\ acid nanofibers for non-invasive physiological signal monitoring. Journal of Materials Chemistry B, 5 (35), \\ 7352-7359. \\ Authors \\ Ayesha Sultana, Sujoy Ghosh, Vitor Sencadas, Tian Zheng, Michael J. Higgins, Tapas Middya, and \\ Dipankar Mandal
}

This journal article is available at Research Online: https://ro.uow.edu.au/aiimpapers/2751 


\section{Human skin interactive self-powered wearable piezoelectric bio-e-skin by electrospun poly-l-lactic acid nanofibers for non-invasive physiological signal monitoring}

Ayesha Sultana ${ }^{\dagger}$, Sujoy Kumar Ghosh ${ }^{\dagger}$, Vitor Sencadas ${ }^{\ddagger, \#}$, Tian Zheng ${ }^{\mathrm{a}}$, Michael Higgins ${ }^{\mathrm{a}}$, Tapas Ranjan Middya ${ }^{\dagger}$, Dipankar Mandal ${ }^{\dagger, *}$

${ }^{\dagger}$ Organic Nano-Piezoelectric Device Laboratory, Department of Physics, Jadavpur University, Kolkata 700032, India

${ }^{\ddagger}$ Australian Centre of Excellence for Electromaterials Science (ACES), University of Wollongong, NSW 2522, Australia

\#School of Mechanical, Materials and Mechatronics Engineering, University of Wollongong, Wollongong, NSW 2522, Australia

${ }^{a} \mathrm{ARC}$ Centre of Excellence for Electromaterials Science, Intelligent Polymer Research Institute/AIIM Faculty, Innovation Campus, University of Wollongong, Squires Way, NSW, Australia

*Corresponding Author

Dr. Dipankar Mandal

E-mail:dipankar@phys.jdvu.ac.in;dpkrmandal@gmail.com

Fax: +91-33-2413-8917

Tel.: $+913324146666 \times 2880$ 


\begin{abstract}
Flexible and wearable piezoelectric bio $e$-skin (PBio-e-skin) based on electrospun poly(Llactide) PLLA nanofiber membrane is demonstrated for human physiological signal monitoring and detecting dynamic tactile stimuli. The molecular orientations of the $\mathrm{C}=\mathrm{O}$ dipoles by electrospinning technique results in a longitudinal piezoelectric charge coefficient, $d_{33}$ value of $\sim(3 \pm 1) \mathrm{pm} / \mathrm{V}$ realized by piezoresponse force microscopy, allow the PBio-e-skin for pressure sensing applications. The robust mechanical strength (Young's modulus $~ 50 \mathrm{MPa}$ ) of nanofiber membrane ensures PBio-e-skin's operational stability (over 375,000 cycles). Owing to the superior mechanical sensitivity of $\sim 22 \mathrm{~V} / \mathrm{N}$, PBio-e-skin has the ability to measure subtle movement of muscle in the internal organs such as esophagus, trachea, motion of joints and arterial pressure by recognition of strains on human skin. This flexible and light weight PBio-e-skin can be valuable for monitoring vital signs in detecting cardiovascular diseases, assessing health status and, also for voice recognition. Thus, the low cost, environmental friendly PBio-e-skin can be used for non-invasive, artery pulse wave monitoring, which may lead to the use of flexible PBio-e-skin in health monitoring and diagnostics incardiovascular medicine.
\end{abstract}

DOI: 10.1038

Keywords: piezoelectric, PLLA, electrospinning, $e$-skin, flexible, human motion detection, wearable, nanofiber, health monitoring

\title{
Introduction
}

Skin-mountable, stretchable and wearable/epidermal electronic devices for human motion detection, ${ }^{1,2}$ flexible electronic skin $(e$-skin $),{ }^{3}$ human physiological and behavioural signal detection, ${ }^{4,5}$ human body temperature monitoring ${ }^{6}$ and personal healthcare, ${ }^{7}$ have recently received enormous attention due to their facile interaction with skin or organs of the human body. These flexible sensors can be attached into clothing or directly mounted on the human 
skin for the real-time monitoring of human activities and physiological measurements with high efficiency. They must fulfil various minimum necessities including large stretchability, lightweight, flexibility, durability, biocompatibility for stable contact with human skin with minimal irritation.

So far design and development of wearable sensor systems for measuring and quantifying physical signals are based on force-driven changes in resistivity, ${ }^{8,9}$ capacitance, ${ }^{10,11}$ piezoand tribo-electricity. ${ }^{12,13}$ In spite of the possibility and superior performance, wide spread applications of some of these methods is perhaps limited by structure complexity, sensitivity, dependence on external power source and fabrication of high-quality materials. ${ }^{14}$ One confront for making pressure sensors that meet the aforementioned demands of high stretchability, ultrahigh sensitivity and biocompatibility is to use piezoelectric nanofiber membranes. In this context, poly(L-lactide) (PLLA) is the one which is biocompatible, biodegradable, optically active polymer and continuously drawing increasing attention due to its medical usefulness in tissue engineering scaffolds. ${ }^{15-17}$ Many biological systems exhibit unique uniaxial symmetry where both direct and inverse piezoelectric effects are present but only $d_{14}$ and $d_{25}$ (with same magnitude and opposite sign) shear piezoelectric constants are finite. PLLA molecular chain consists of chiral molecules where $-\mathrm{CO}-\mathrm{O}$ polar group joined to an asymmetric carbon atom and oriented in helical conformation in the molecules. When the helix is sheared through its side chain $-\mathrm{C}=\mathrm{O}$ dipole makes a slight rotation that changes the polarization of the chain molecule. ${ }^{18,15}$ Polarization appears in a direction perpendicular to the plane of applied stress. The sum of these rotation results in electric polarization. ${ }^{19,15}$ Owing to its bio- piezoelectricity, PLLA is a suitable material for in-vivo applications where biomaterial based self-powered system is particularly relevant. ${ }^{20,21}$ In previous, several biomaterials such as, collagen, chitin, cellulose, gelatin, M13 bacteriophage and many others 
possessing longitudinal piezoelectric coefficient $\left(\mathrm{d}_{33}\right)$ have been considered as engineering effective materials for developing self-powered systems. ${ }^{22-26}$

The thermodynamically stable conformation of PLLA is the $\alpha$-crystalline form, where the $\mathrm{C}=\mathrm{O}$ dipoles are randomly oriented along the main chain, resulting non-polarity of PLLA in this form. In order to induce piezoelectricity the chains must be stretched to transform the $\alpha$ form into $\beta$-crystalline form where random orientation of molecular chains is aligned along

stretched direction. ${ }^{27}$ The electrospinning process applies stretching force into the molecular chains of PLLA under high electric field and thus aligns molecular dipoles resulting net polarization, in a single step. ${ }^{28}$

Herein, a simple structured, easy to fabricate, wearable, lightweight, cost-effective piezoelectric bio-e-skin was prepared for pressure monitoring. This device is capable of working in a multimodal manner for human physiological signal monitoring, sports performance monitoring and also for voice recognition. It asserts a sensitivity of $22 \mathrm{~V} / \mathrm{N}$ as well as a pressure detection extent down to $18 \mathrm{~Pa}$ for detection of feather light pressure. In loading-unloading cycle a negligible change in output signal was experimentally noticed over 375,000 cycles, suggesting its performance stability and durability. The flexible $e$-skin thus evidenced the capability of monitoring human physiological signals such as throat and wrist pulse and muscle movement when a person is speaking, which might extend their possible applications for real-time disease diagnosis and voice recognition.

\section{Experimental Section}

Materials. Poly (L-lactic acid) (PLLA, Purasorb PL18, $M_{w}=217-225 \mathrm{kDa}$ ) from Corbion (Netherlands), N,N-dimethylformamide (DMF, from Merck), dichloromethane (MC, from 
Sigma-Aldrich), Copper ( $\mathrm{Cu}$ )-nickel (Ni) plated fine knit polyester fabric (Coatex Industries, India) and poly(dimethylsiloxane) (PDMS) (SYLGARD, 184 SILICONE ELASTOMER).

PLLA electrospun membranes preparation: PLLA was dissolved in a mixed solvent of DMF and dichloromethane $(3 / 7 \mathrm{v} / \mathrm{v})$ to achieve a polymer concentration of $10 \mathrm{wt} \%$ of the solution. The polymer solution was dissolved at room temperature with the help of a magnetic stirrer until complete polymer dissolution. ${ }^{29}$ The polymer solution was placed in a commercial glass syringe $(10 \mathrm{~mL})$ fitted with a steel needle with $500 \mu \mathrm{m}$ of inner diameter. Electrospinning was conducted at $1.25 \mathrm{kV} . \mathrm{cm}^{-1}$ with a high voltage power supply from Gamma High Voltage. A syringe pump (from KD Scientific) was used to feed the polymer solutions into the needle tip at $0.5 \mathrm{~mL} \cdot \mathrm{h}^{-1}$. The electrospun fibres were collected in ground collecting plate placed at $20 \mathrm{~cm}$ apart from the needle. All experiments were conducted at 21 $\pm 2{ }^{\circ} \mathrm{C}$ and a relative humidity of $43 \pm 5 \%$.

Piezoelectric bio-e-skin (PBio-e-skin) fabrication. The simple structured PBio-e-skin was fabricated by the following method. At first a $(2.5 \times 2) \mathrm{cm}^{2}$ area was cut from electrospun PLLA membrane with thickness of $\sim 40 \mu \mathrm{m}$. Then top and bottom electrodes were prepared by attaching $\mathrm{Ni}-\mathrm{Cu}-\mathrm{Ni}$ plated polyester fabric on either side with effective contact area of (2 $\times 1.5) \mathrm{cm}^{2}$. Then conducting copper wires were attached on both electrodes. Finally, it was sandwiched between two layers (thickness of $\sim 0.5 \mathrm{~mm}$ ) of) PDMS which were prepared by 10:1 PDMS curing agent ratio and cured at $60^{\circ} \mathrm{C}$ for $2 \mathrm{~h}$.

\section{Characterization}

The morphological features of the electrospun fibers were imaged by a Field Emission Scanning Electron Microscope (FE-SEM, FEI, INSPECT F50) operated at an acceleration voltage of $20 \mathrm{kV}$. Fourier transform infrared (FT-IR) spectra were recorded (Bruker Tensor 
II) to characterizethe different bands within the NFs. The topography images were obtained in AC mode with an MFP3D atomic force microscope (AFM) (Asylum Research, CA). Scan rate was set as $0.5 \mathrm{~Hz}$. Dual AC Resonance Tracking Mode piezoresponse force microscopy (PFM) was used for measuring piezoresponse locally. A Pt/Ir coated silicon tip with force constant of $2.8 \mathrm{~N} / \mathrm{m}$ and gold layer on the substrate was used as top and bottom electrodes. Contact frequency varied from $320 \mathrm{kHz}$ to $340 \mathrm{kHz}$ due to slightly changes on sample surface from point to point. A small AC voltage $(200 \mathrm{mV})$ was applied to oscillate the tip during measurement. A sweeping DC bias (Frequency $=0.2 \mathrm{~Hz}$ ) in the range of $\pm 25 \mathrm{~V}$ was applied to the tip. 5 cycles of sweeping triangle/square waves (frequency $=0.2 \mathrm{~Hz}$ ) were applied to the tip to offer the fiber with essential electric field. Igor Pro 6.36 Software was used to obtain the butterfly loops. Stress-strain measurement was carried out using universal testing machine (Tinius Olsen H50KS). PBio-e-skin was fabricated by simply sandwiching the nanofiber mat between two conducting $\mathrm{Ni}-\mathrm{Cu}-\mathrm{Ni}$ plated polyester fabric substrates. Electrical contacts for recording open-circuit voltagewere made by $\mathrm{Cu}$-wire leads from PBioe-skin connected through a National Instrument (NI) Data acquisition (DAQ) device (NI, USB 6000) using a sampling rate of 1000 samplesper second, interfaced with a computer with a standaloneprogram made by using LabVIEW software and current measurement were carried out with KEITHLEY 6485 picoammeter.

\section{Results and Discussion}

The schematic diagram in Fig. 1a shows a PLLA nanofiber fabrication process by electrospining technique. The resulting PLLA nanofiber mat is used in flexible PBio-e-skin $\left(2 \times 1.5 \mathrm{~cm}^{2}\right)$ design with splendid capability to bend in desirable shape (Fig. 1b). The morphology of as-electrospun nanofibers of PLLA is shown in Fig. 1c. The nanofibers are entangled with one another and form a network-structured fiber mat. All fibers evidence 
smooth and bead free morphology with an average fiber diameter found to be $450 \mathrm{~nm}$ (inset of Fig. 1c). Fig. 1d shows the FTIR spectra of nanofibers. The bands at 871, 1088, 1184, $1755 \mathrm{~cm}^{-1}$ are assigned to the $\mathrm{C}-\mathrm{C}$ backbone stretching, symmetric and asymmetric stretching of $\mathrm{C}-\mathrm{O}-\mathrm{C}$ group and stretching vibration of $\mathrm{C}=\mathrm{O}$ which are responsible for piezo- and ferroelectricty in PLLA nanofibers. ${ }^{30,31}$ All the bands of different wavenumbers are labelled in Fig. 1d.

As the piezoelectric output performance of the nanofibers is greatly affected by their mechanical properties in addition to piezoelectric coefficient value, thus, tensile stress-strain behaviour of PLLA nanofibers has been studied as shown in Fig. 2a. Firstly, when a small external stress $(<8 \mathrm{kPa})$ is applied, the non-woven nanofibers are forced to align along stress direction. This forced orientation results in a nonlinear elastic behaviour. With continual increase of stress, the stress-strain curve exhibits a linear elasticity. Thus, the PLLA fibers have ultimate tensile stress of $6.8 \mathrm{MPa}$, tensile strain of $42.3 \%$ with Young's modulus value of $\sim 50 \mathrm{MPa}$. PLLA nanofiber membrane with the robust mechanical strength could be applied as promising material towards real time applications. 

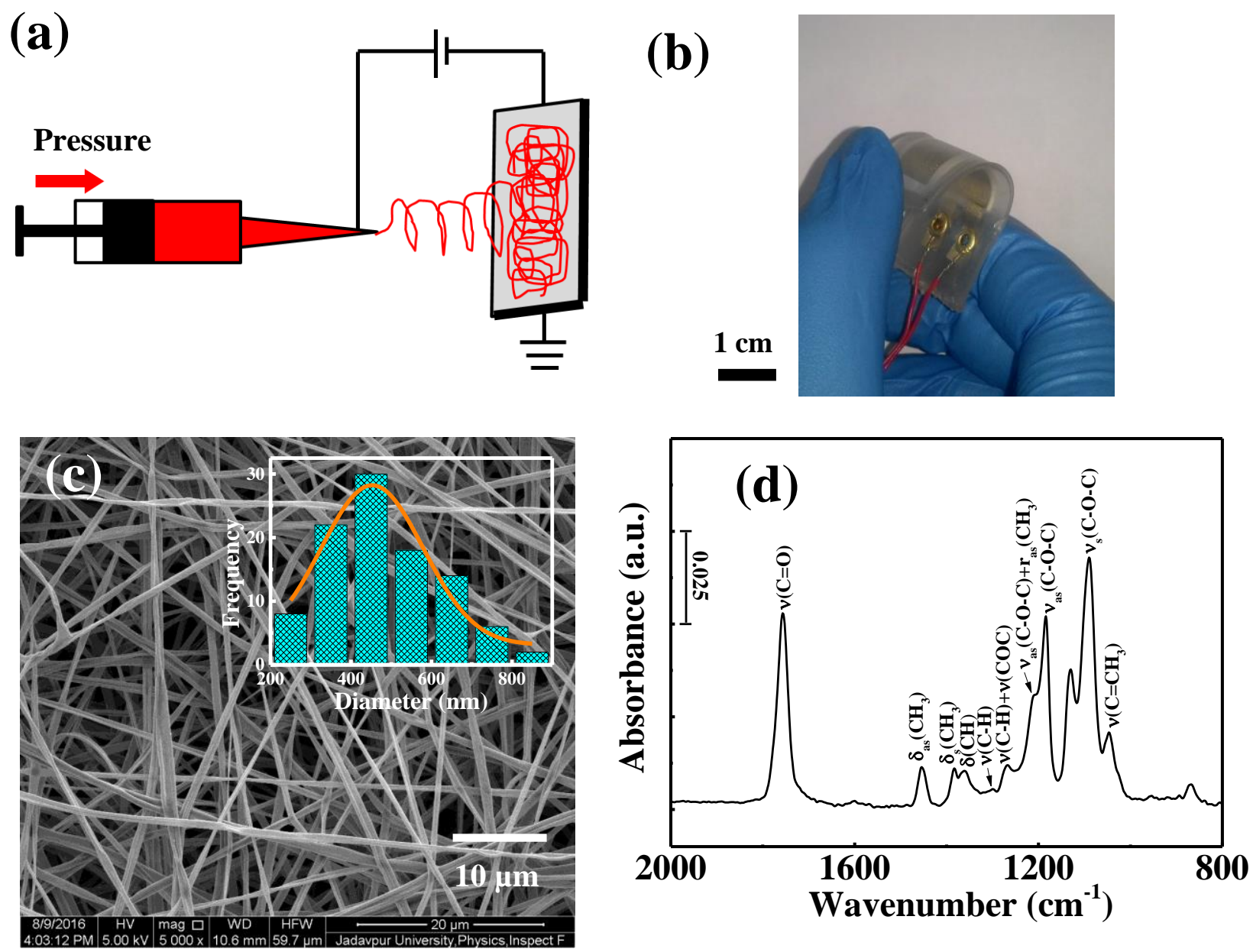

Fig. 1 (a) Schematic illustration of the experimental setup for preparation of PLLA nanofibers. (b) The digital photograph of fabricated PBio-e-skin from PLLA nanofiber mat showing its flexibility by human fingers. (c) FE-SEM micrograph and corresponding fiber diameter distribution plot in the inset. (d) FT-IR spectra from 2000 to $800 \mathrm{~cm}^{-1}$ frequency region.

To quantify local piezo- and ferro-electricity of PLLA nanofiber piezoelectric hysteresis loops composed of phase voltage and amplitude voltage loops, PFM was conducted in a single polymeric fiber deposited on a gold substrate. It assesses the dipole orientation and 
mechanical fashion of the nanofiber when an electrical signal was applied by a metal-coated AFM tip and scanning the sample.
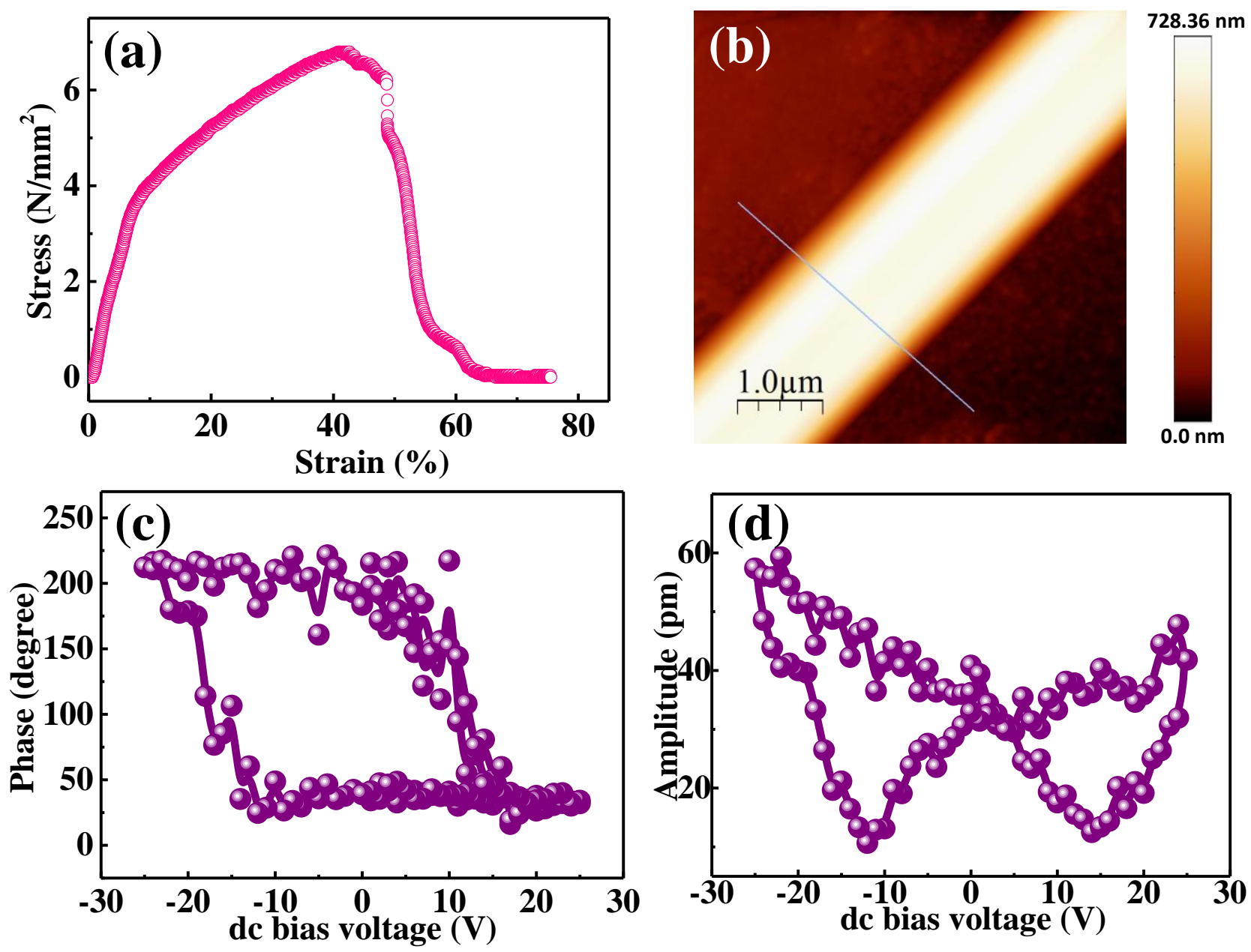

Fig. 2 (a) Stress-strain curve, (b) AFM topography, (c) PFM phase-voltage and (d) amplitude-voltage hysteresis loops of the PLLA nanofibers.

The AFM topography investigation (Fig. 2b) shows that the PLLA nanofiber has height of $650 \mathrm{~nm}$ (supporting information, Fig. S1). Excellent phase and amplitude responses (Fig. 2cd) from one single nanofiber have been observed as a function of applied voltage between $25 \mathrm{~V}$ to $+25 \mathrm{~V}$ to the AFM conductive cantilever tip with respect to ground. The PFM phase response demonstrates a clear and almost rectangular hysteresis in the phase versus voltage 
diagram (Fig. 2c), with a phase contrast of $180^{\circ}$ at bias voltage of $25 \mathrm{~V}$. In response to sweeping DC voltage the hysteretic switching of the phase signal by $180^{\circ}$ is assigned to the switching of the direction of polarization of the $\mathrm{C}=\mathrm{O}$ dipoles, which implies the evidence of nano-scale ferroelectricity of the PLLA nanofibers. The PFM amplitude response is hysteretic and the shape resembles the piezoelectric "butterfly loop" (Fig. 2d). Each point of this loop includes information about piezoelectric deformation $\left(\varepsilon_{\mathrm{p}}=\mathrm{d}_{33} \mathrm{E}\right.$, where $\mathrm{d}_{33}$ is the longitudinal piezoelectric co-efficient) under corresponding applied voltage/electric field (E). The estimated effective piezoelectric coefficient from the slope of the curves of amplitude versus voltage is $d_{33} \sim 3 \pm 1 \mathrm{pm} / \mathrm{V} .^{32,33}$ The observations of hysteretic phase switching and butterfly shaped amplitude loops reveal a clear evidence of ferro- and piezo-electric properties in the PLLA nanofiber mat.

Thus, we have fabricated PLLA nanofibers based piezoelectric bio $e$-skin (PBio-e-skin) which could also be used for mechanical energy harvesting and piezotronics applications. The performance of the PBio-e-skin as a function of various deformation frequencies was investigated since mechanical energies from environment is irregular and varies in frequency. The corresponding output voltage and current are shown in Fig. 3a-b. As the frequency of the applied stress $(\sim 0.3 \mathrm{MPa})$ increases, the open circuit voltage $\left(\mathrm{V}_{\mathrm{oc}}\right)$ increases because the electrons are flowing to reach equilibrium in a shorter time. ${ }^{34}$ 


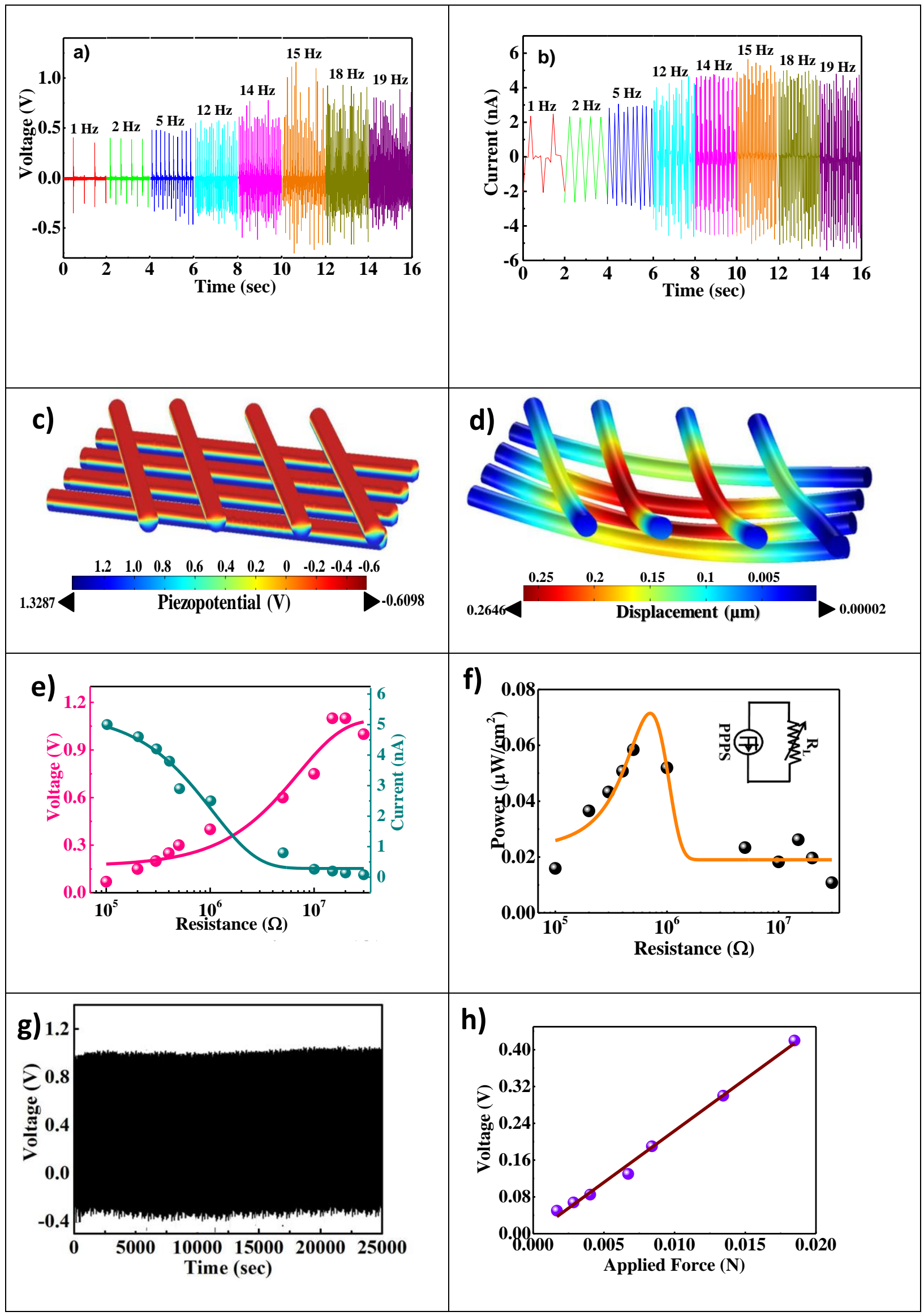


Fig. 3 Characterization of output performance of the nanogenerator under external force with different frequencies in terms of (a) voltage and (b) current in the range of 1 to $19 \mathrm{~Hz}$. (c) Simulated piezopotential distribution of the network structure PLLA nanofibers. (d) Simulated deformation distribution of network structured PLLA nanofibers at the constant external stress amplitude of $0.3 \mathrm{MPa}$. (e) Variation of output voltage and current as well as (f) instantaneous output power density as a function of variable external resistances ranging from $100 \mathrm{k} \Omega-30 \mathrm{M} \Omega$. Inset shows the corresponding equivalent circuit diagram. (g) The stability test of the PBio-e-skin under a $0.3 \mathrm{MPa}$ stress by recording the open circuit voltage as a function of time over 375,000 cycles. (h) Piezoelectric output performance of the PBio-e-skin under different applied forces.

The maximum $\mathrm{V}_{\mathrm{oc}}$ reaches up to $1 \mathrm{~V}$ at the frequency of $15 \mathrm{~Hz}$ then it gradually decreases to $0.7 \mathrm{~V}$ at $19 \mathrm{~Hz}$. The short-circuit current $\left(\mathrm{I}_{\mathrm{sc}}\right)$ presents an increasing trend with the increase of frequency, from $2.2 \mathrm{nA}$ at $1 \mathrm{~Hz}$ to $5 \mathrm{nA}$ at $15 \mathrm{~Hz}$ (Fig. 3b), because the deformation rate increases with applied frequency, which leads to a higher flow rate of charges, that is, higher current. As a matter of fact, when the compressive force is of high frequency the nanogenerator then cannot retrieve the original posture before next impact of force and thus $\mathrm{V}_{\mathrm{oc}}$ and $\mathrm{I}_{\mathrm{sc}}$ decreases at very high frequency. ${ }^{35}$

To yield a quantitative explanation on the piezopotential distribution inside the deformed PLLA nano-fibers based nanogenerator, theoretical simulation was conducted using the Finite Element Method (FEM) by COMSOL multiphysics software under uniaxial $\sigma_{\mathrm{a}} \sim 0.3$ MPa (Fig. 3c). It is demonstrated by eight nanofibers based network structure. The experimentally measured piezopotential $(\sim 1.0 \mathrm{~V})$ was very much consisted with the simulated potential difference of $1.2 \mathrm{~V}$. The resulting deformation (i.e., maximum displacement $\sim 0.2$ $\mu \mathrm{m})$ distribution of nano-fibers corresponding to the piezopotential distribution is shown in 
Fig. 3d. Interesting fact is that, maximum deformations $(\sim 0.2 \mu \mathrm{m})$ occur in the interaction region between lower and upper layer of the fibers during applied stress. In the noninteractive regions, the deformations gradually diminish. It indicates that the networkstructure of nanofibers enhance the resulting polarization in comparison to single fiber due to cooperative electromechanical mutual interaction among the adjacent fibers during applied pressure.

Eventually, the PBio-e-skin is useful to drive portable electronic devices by harvesting small scale mechanical energies. To assess the output power performance, the PBio-e-skin is directly connected to the loads of different resistances $\left(\mathrm{R}_{\mathrm{L}}\right)$ ranging from $100 \mathrm{k} \Omega$ to $30 \mathrm{M} \Omega$, (under a frequency of $15 \mathrm{~Hz}$ ). The voltage across the load increases and saturates to open circuit voltage when the resistance gets infinitely large. In contrast, the current through the load decrease from short circuit current when the resistance increases (Fig. 3e). The instantaneous output power $(\mathrm{P})$ was calculated from the relation, $\mathrm{P}=\mathrm{V}^{2} / \mathrm{A} \cdot \mathrm{R}_{\mathrm{L}}$, where, $\mathrm{A}$ is the effective contact area, and $\mathrm{V}$ is the voltage drop across $\mathrm{R}_{\mathrm{L}}$. The dependency of output power with load resistance is shown in Fig. 3f, where maximum value of $0.07 \mu \mathrm{W} / \mathrm{cm}^{2}$ at a load resistance of $700 \mathrm{k} \Omega$ is achieved. ${ }^{36,37}$

In addition, to further investigate the long-term stability of the PBio-e-skin under mechanical deformation, a pressure of $0.3 \mathrm{MPa}$ with a frequency of $15 \mathrm{~Hz}$ was applied. The fatigue test of the PBio-e-skin was done by recording the output voltage as a function of time over extended cycling times $(375,000$ cycles, Fig. $3 \mathrm{~g})$, that indicates the superb stability in the output performance without any electrical output degradation. The voltage amplitudes exhibit negligible changes after a total 375,000 cycles, revealing a high repeatability, durability and stability of the PBio-e-skin. 
To evaluate the sensitivity, the output voltages from the PBio-e-skin have been obtained under different forces. Interestingly, $\mathrm{V}_{\mathrm{oc}}$ changes linearly with the applied force. Quantitatively, the sensitivity is defined as, $\mathrm{S}=\frac{\Delta V_{o c}}{\Delta \sigma_{a}}$, where $\Delta V_{o c}$ and $\Delta \sigma_{a}$ are the differences of $\mathrm{V}_{\mathrm{oc}}$ and $\sigma_{\mathrm{a}}$ respectively. To understand the relationship between applied force and piezoelectric output voltage, different weights was dropped from a height of $10 \mathrm{~cm}$. The force from different weight (supporting information, Text S1) corresponds to distinct output voltage. Fig. 3h shows a nearly linear relationship between the applied force and output voltage. Interestingly, from the slope of the linear fitted data the sensitivity of the PBio-e-skin is found to be $22 \mathrm{~V} / \mathrm{N}$.

(a) $\bigcirc \mathrm{cO} \bigcirc \mathrm{H}$

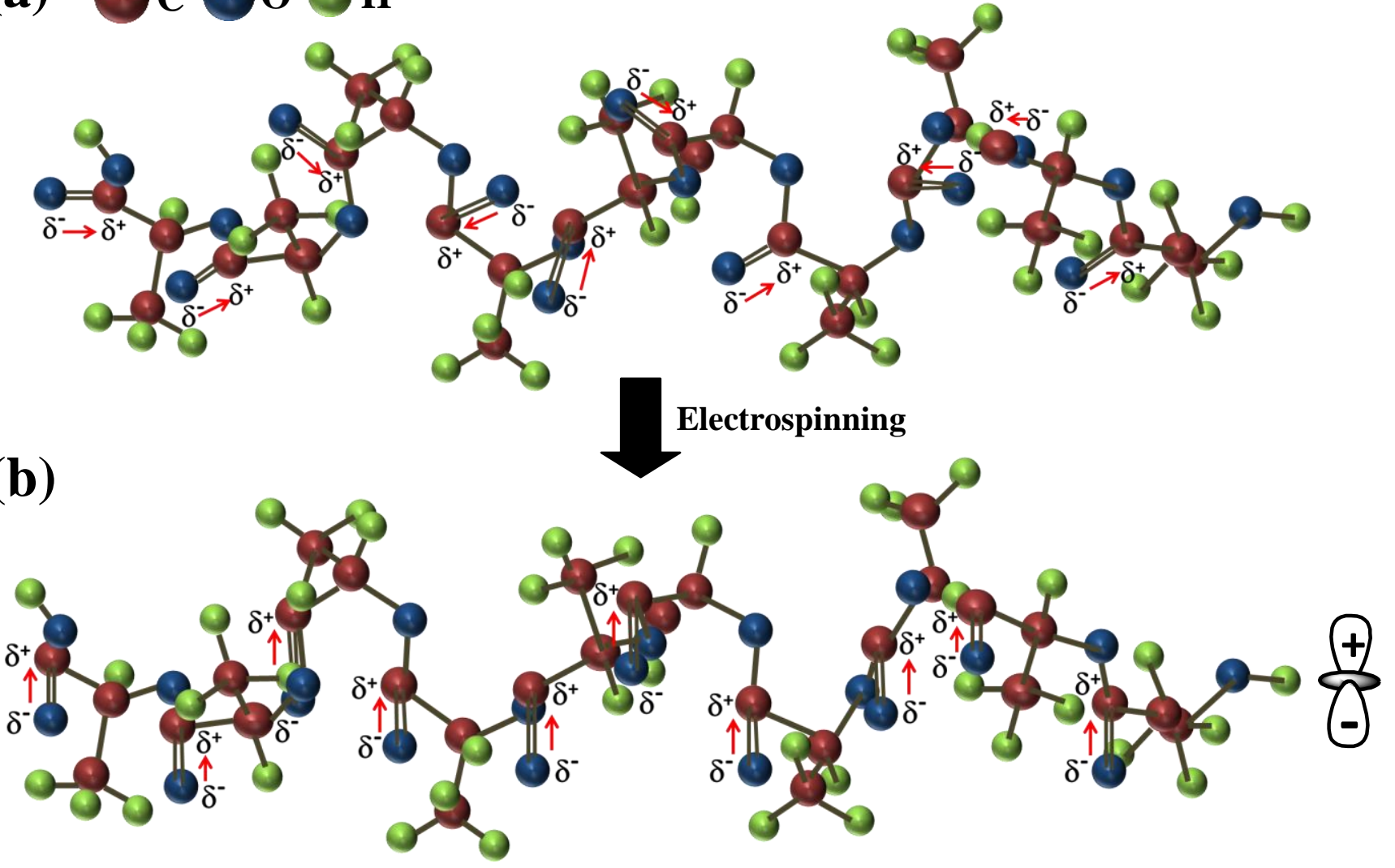


Fig. 4 (a) Molecular structure of PLLA chain with orientation of $\mathrm{C}=\mathrm{O}$ dipoles in all directions. (b) Preferential orientation of the $\mathrm{C}=\mathrm{O}$ dipoles due to electrospinning process.

The origin of piezoelectricity in biomaterials is not absolutely disclosed as they do not follow the classic models of piezoelectric theories. PLLA exhibit shear piezoelectricity, in contrast, a collection of ordered PLLA nanofiber existing in PBio-e-skin, exhibits a completely different behavior. This kind of difference was also being observed formerly in M13 bacteriophage [26]. In this work, the strong electric field used during electrospinning results in stretching along the longitudinal direction of the nanofiber and thus the randomly oriented $\mathrm{C}=\mathrm{O}$ dipoles (Fig. 4a) are aligned perpendicular to nanofiber length (Fig. 4b), and the high sensitivity of the PBio-e-skin is due to such alignment of the dipoles. Additionally, the other possible explanation for such enhancement of the PBio-e-skin is associated to the cooperative electromechanical interaction among the adjacent nanofibers during the applied mechanical pressure in contrast of a single nanofiber. ${ }^{22,26,38}$

Furthermore, the PBio-e-skin displays a sensitive response to external impact with extremely lightweight objects. The experiment was demonstrated with a feather $(0.2 \mathrm{~g})$. It detects the external impacts of $45 \mathrm{~Pa}$, and responds with an output response of $0.15 \mathrm{~V}$ (Fig. 5a). Another object lighter than the feather, for example, a cubical block made with thermocol $(0.07 \mathrm{~g})$, was used to investigate the sensitivity of the PBio-e-skin. By detecting the impact of the cube, it exhibited an output response of $0.06 \mathrm{~V}$ (Fig. 5b). Therefore, the output response of the PBio-e-skin upon loading/unloading the two light weight objects, result in promising pressure sensing with detection capability as low as 45 and $18 \mathrm{~Pa}$, respectively. Hence, PBioe-skin is very sensitive and can identify even a small change of mechanical impact accurately, and displays distinct electrical output responses. 

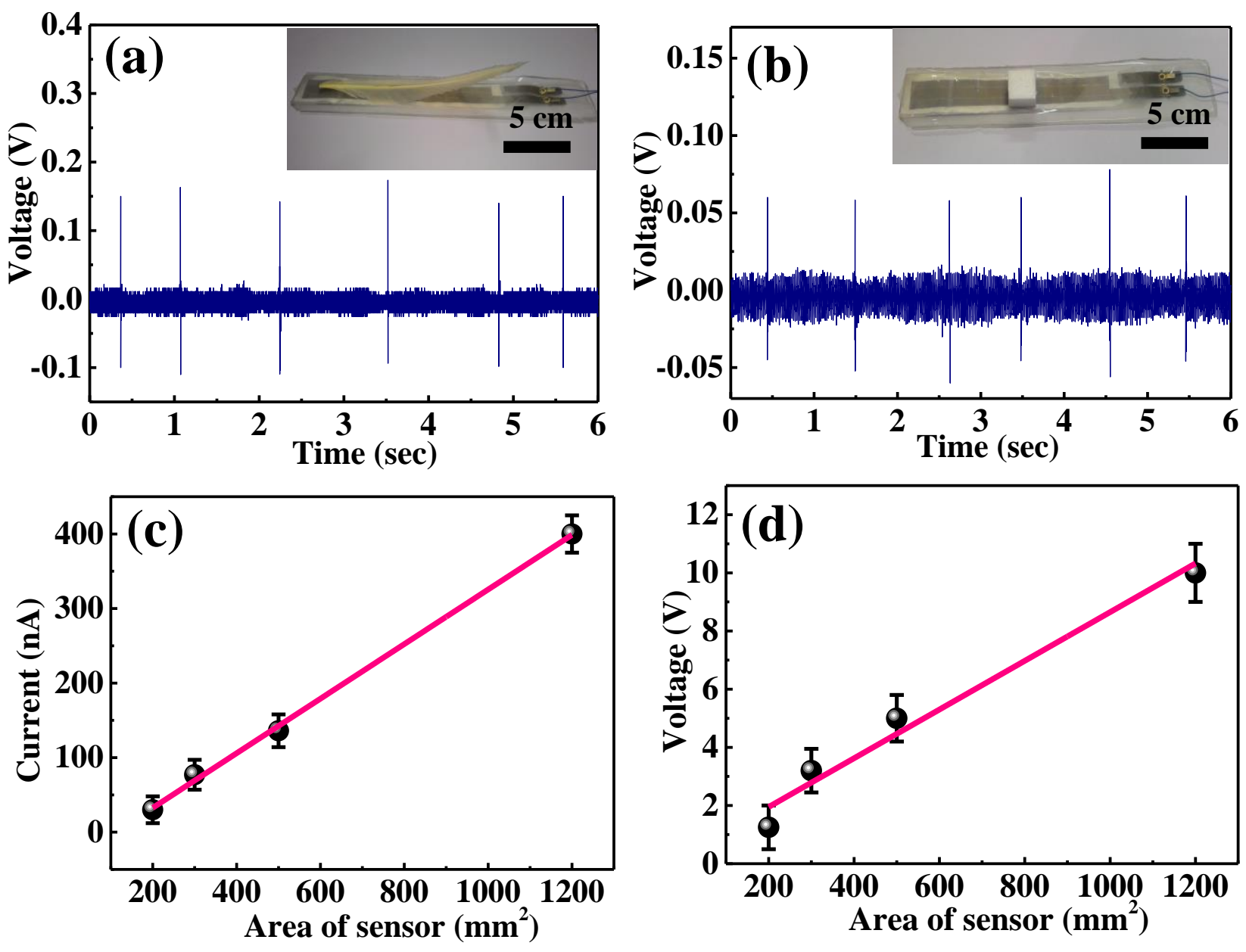

Fig. 5 Output voltage response from PBio-e-skin with different applied external pressure for the detection of a (a) feather (illustrated in the inset) and a (b) cubic thermocol block (illustrated in the inset). Piezoelectric output (c) current and (d) voltage responses as a function of area of PBio-e-skin.

To demonstrate the energy harvesting performance and practical application in rough environment, the PBio-e-skin electrical responses was investigaed by applying compresssive stress periodically by human finger. Here finger was imparted on PBio-e-skin made with 
different areas. It was observed that the $I_{s c}$ and $V_{o c}$ increases from $30 \mathrm{nA}$ to $400 \mathrm{nA}$ (Fig. 5c) and $1.25 \mathrm{~V}$ to $10 \mathrm{~V}$ (Fig. 5d) respectively with an increase in sensor area ranging from 200 to $2200 \mathrm{~mm}^{2}$. The output current and voltage shows almost linear depencency with the area of total active area of the sensor following the relations of the piezoelectric theory. ${ }^{39}$ Thus the current and voltage value can be modulated by changing the active area of PBio-e-skin as per the power requirement of portable electronic devices.

In addition, the PBio-e-skin was further used for detecting static tactile stimuli. To show applicabiliy of PBio-e-skin, as skin mountable sports performance monitoring device the sensor was attatched to the wrist Fig. 6a. The static strain on the PBio-e-skin increases upon bending of wrist, and recovers its original position after straightining of wrist. The response of the PBio-e-skin on bending and relaxing of wrist with good quality of sensitivity is demonstrated in Fig. 6b. This sensory information from skin mountable wearable PBio-e-skin is beneficial for body movement analysis during sports activities and shows the potential of using as epidermal device for skin motion monitoring. ${ }^{40,41}$

The sensor was further placed near the throat and wrist, not only to measure the blood pressure but also for measuring vibrations of muscle movements associated with several human activities. By detecting strain of muscle movement for noninvasively monitoring human activities, the PBio-e-skin was first attatched arround the throat. The PBio-e-skin exhibits high sensitivity to the muscle movement of esophagus (the food pipe) and displays distinct patterns, allowing to differentiate between signals generated by esophagus during drinking and swallowing (Fig. 6c-d). These results show that for monitoring body motions, skin strain monitoring is an effective method.

To demonstrate the performance as a stretchable PBio-e-skin for several biomedical application the PBio-e-skin was attatched to carotid artery and raidal artery of an adult 
human. At first, the sensor is placed on the wrist to allow the measurement of blood pulse wave (Fig. 6e). Three wave related to radial artery pressure are incident blood pulse wave, reflected wave from hand and reflected wave from lower body. The acquired real-time current output response from radial artery pressure is given in Fig. 6f. PBio-e-skin is placed over carotid artery Fig. $6 \mathrm{~g}$ and the real-time current output over several pulse period is shown in Fig. 6h.
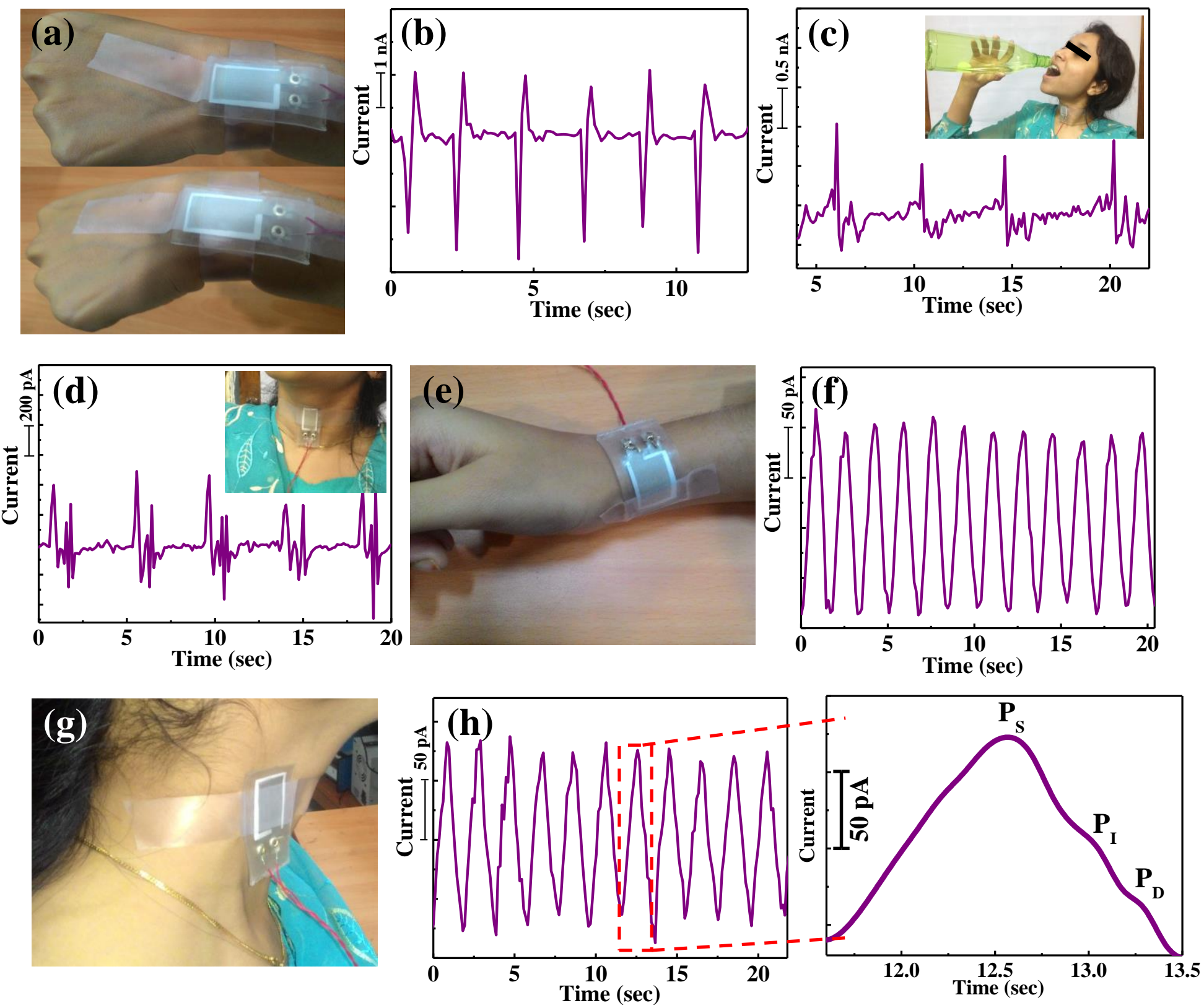
Fig. 6 (a) Photograph of the PBio-e-skin mounted on wrist joint. (b) Detected wrist joint motion in terms of current output. Monitoring of strain caused by muscle movement for function of esophagus during (c) drinking and (d) swallowing. (e) Photograph of the PBio-eskin placed on a wrist for measuring the pressure associated with flow of blood through nearsurface arteries. (f) Current vs time plot for the PBio-e-skin mounted on the wrist. (g) Photograph showing PBio-e-skin directly attached to the carotid artery of an adult subject. (h) The real-time current outputs for the sensor placed over carotid artery. The enlarged view in the right shows the systolic $\left(\mathrm{P}_{\mathrm{S}}\right)$, point of inflexion $\left(\mathrm{P}_{\mathrm{I}}\right)$ and diastolic peak $\left(\mathrm{P}_{\mathrm{D}}\right)$ of one cycle.

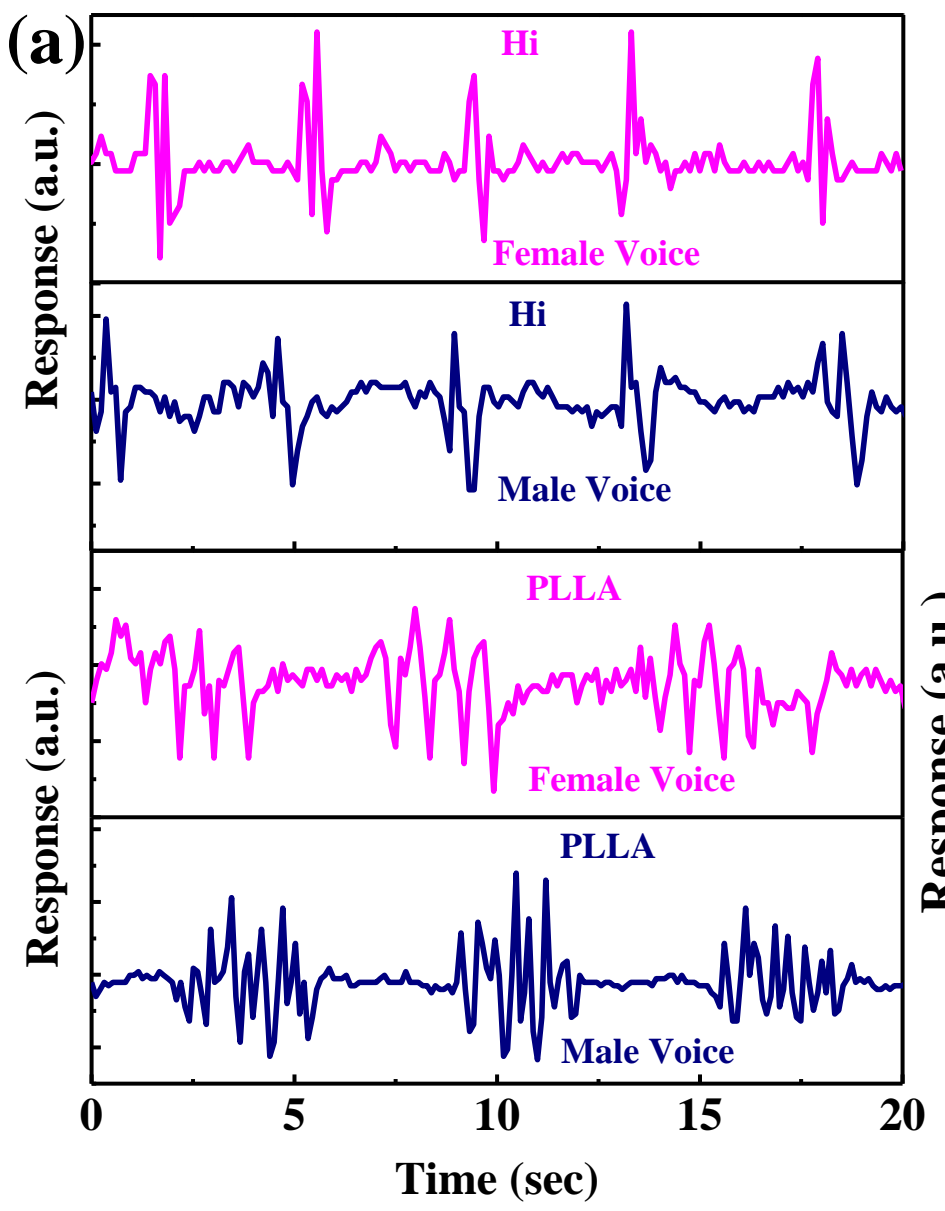

(b)

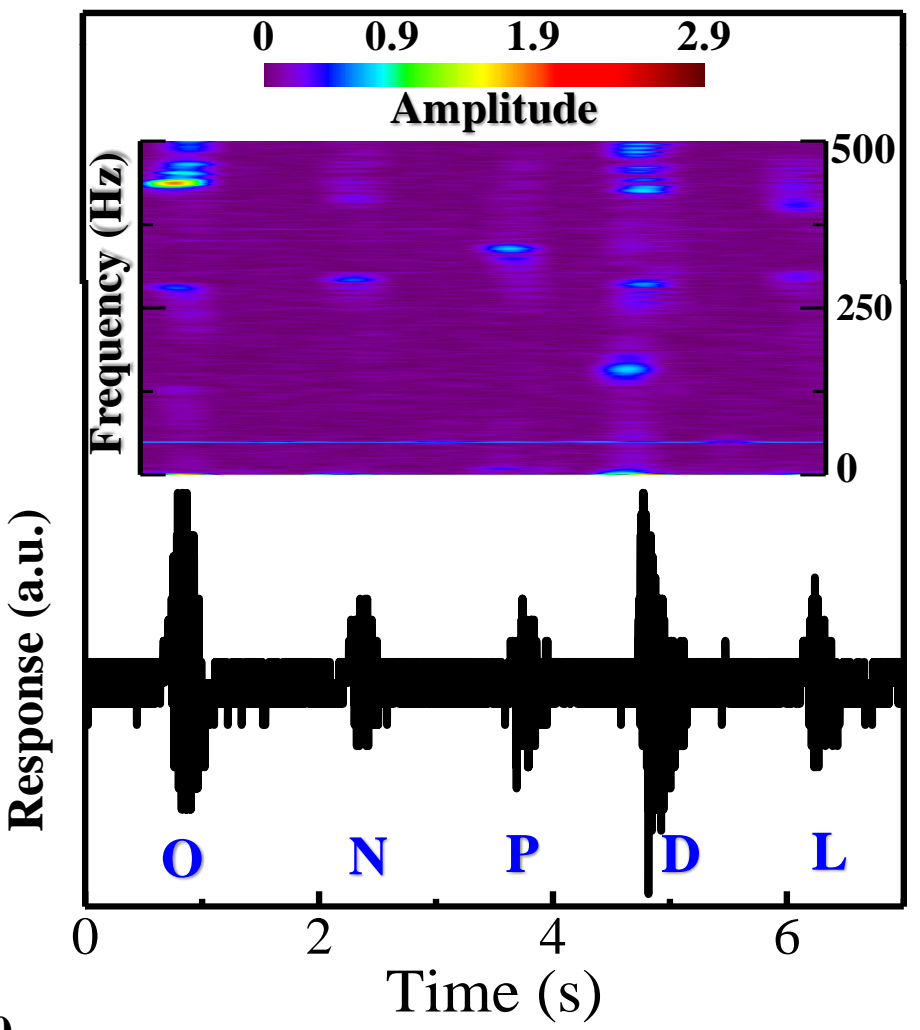


Fig. 7 Wavefront spectrum of female and male voices saying (a) "hi" and "PLLA". (b) Output wavefront spectrum observed from PBio-e-skin as different letters (O, N, P, D and L) are pronounced with FFT signal in the upper part.

Change in current results from variation of pressure related to blood flow. Systolic peak $\left(\mathrm{P}_{\mathrm{S}}\right)$, point of inflexion $\left(\mathrm{P}_{\mathrm{I}}\right)$ and diastolic peak $\left(\mathrm{P}_{\mathrm{D}}\right)$ can be identified from the enlarged image. These peaks are known to be composed of three waves which are resulted from pulse wave ejected from left ventricle, its reflected wave and ejected blood pulse back to left ventricle. ${ }^{42}$ These types of pulse waveforms provide valuable information for diagnosis and therapy for cardiovascular diseases. ${ }^{43,44}$ The skin mounted sensor developed here, proved to be useful as an arterial pulse wave monitor.

As a speech pattern recognition system, the PBio-e-skin was attached firmly to the human speaker's neck and pressure difference of muscle movement during speech was recorded. The sensor exhibits separate patterns when the different words such as "hi" and "PLLA" (Fig. 7a) were spoken. Both these words were recorded for several cycles to investigate its repeatability and similar characteristic patterns were obtained. In addition, it can also differentiate between female and male voice, accurately. For non-invasively monitor the variation of epidermis deformation and muscle movement throughout the throat during phonation, alphabets such as 'O', 'N', 'P', 'D' and 'L' were pronounced. PBio-e-skin can clearly detect and distinguish between the pronunciation of alphabets by mimicking the vocal cord vibration and generating output signal waveform (Fig. 7b). The corresponding short time Fourier transforms (STFT) processed spectrograms of each alphabet (upper part of Fig. 7b) shows different output profiles. It is evident from the spectrogram that the maximum amplitude of ' $\mathrm{O}$ ' is in the higher frequency range of $400 \mathrm{~Hz}$, for the letters $\mathrm{N}$ ' and ' $\mathrm{P}$ ', the frequency range is around $250 \mathrm{~Hz}$, and for ' $\mathrm{D}$ ' it is from 100-500 Hz. These results provide 
interesting method for using the PBio-e-skin to monitor muscle movement, voice recognition and assisting in speech rehabilitation training.

Thus, the PBio-e-skin can be used as multimodal manner for arterial pulse wave, muscle movement monitoring and as well as voice recognition. This skin strain monitoring might have future applications on biomedical fields and remote control of human/machine interfaces.

\section{Conclusion}

In summary, a stretchable, skin-mountable, highly sensitive, multifunctional piezoelectric bio $e$-skin was developed by using PLLA nanofiber membrane with a longitudinal piezoelectric coefficient value of $3 \pm 1 \mathrm{pm} / \mathrm{V}$. Systematic and elaborate strain tests for sensor and energy devices based on the fabricated multimodal $e$-skin showed high sensitivity, good stability, and reliability with a detection limit of the device as low as $18 \mathrm{~Pa}$. Skin motion detection was performed byattaching the $e$-skin on different parts of the body for monitoring human physiological signals, such as voice recognition, real-time wrist pulse detection etc. The $e$ skin, with its ability to conform to bend can be used in different applications in a multitude of fields, including personal health monitoring, epidermal electronic device, soft robotics, artificial skins, and human machine interfaces. The good sensing performance of the PBio-eskin broadens its application in cost effective wearable electronics for the prevention of sicknesses, in vivo and in vitro diannostics and the statement of early diseases.

\section{Acknowledgements}

This work was supported by the Science and Engineering Research Board (SERB/1759/2014- 
15), Govt. of India. Ayesha Sultana is supported by Maulana Azad National Fellowship (F117.1/2015-16/MANF-2015-17-WES-53885/(SA-III/Website)) from UGC. Md. Mehebub Alam is supported by UGC-BSR fellowship (Ref. No.P-1/RS/191/14). Authors are also thankful for instrumental facilities developed by DST, Govt. of India under FIST-II programme.

\section{References}

[1] Morteza Amjadi, Aekachan Pichitpajongkit, Sangjun Lee, Seunghwa Ryu, and Inkyu

Park. Highly Stretchable and Sensitive Strain Sensor Based on Silver Nanowire_Elastomer Nanocomposite. ACS Nano, 2014, 8, 5154-5163.

[2] Yan Wang, Li Wang, Tingting Yang, Xiao Li, XiaobeiZang, Miao Zhu, Kunlin Wang, Dehai Wu, and Hongwei Zhu. Wearable and Highly Sensitive Graphene Strain Sensors forHuman Motion Monitoring. Adv. Funct. Mater.2014, 24, 4666-4670.

[3] Chuan Wang, David Hwang, Zhibin Yu, Kuniharu Takei, Junwoo Park, Teresa Chen, Biwu Ma and Ali Javey. User-interactive electronic skin for instantaneous pressure visualization. Nat. Mater. 2013, 12, 899-904.

[4] Xuewen Wang, Yang Gu , Zuoping Xiong, Zheng Cui , and Ting Zhang. Silk-Molded Flexible, Ultrasensitive, and Highly Stable Electronic Skin for Monitoring Human Physiological Signals. Adv. Mater. 2014, 26, 1336-1342.

[5] Joseph T. Muth, Daniel M. Vogt, Ryan L. Truby, Yig itMengüç, David B. Kolesky, Robert J. Wood and Jennifer A. Lewis.Embedded 3D Printing of Strain Sensors within Highly Stretchable Elastomers. Adv. Mater. 2014, 26, 6307-6312. 
[6] R. Chad Webb, Andrew P. Bonifas, Alex Behnaz, Yihui Zhang, Ki Jun Yu, Huanyu Cheng, Mingxing Shi, Zuguang Bian, Zhuangjian Liu, Yun-Soung Kim, Woon-Hong Yeo, Jae Suk Park, Jizhou Song, Yuhang Li, Yonggang Huang, Alexander M. Gorbach and John A. Rogers. Ultrathin conformal devices for precise and continuous thermal characterization of human skin. Nat. Mater. 2013, 12, 938-944.

[7] Tran Quang Trung and Nae-Eung Lee. Flexible and Stretchable Physical Sensor Integrated Platforms for Wearable Human-Activity Monitoring and Personal Healthcare. Adv. Mater. 2016, 28, 4338-4372.

[8] Takeo Yamada, Yuhei Hayamizu, Yuki Yamamoto, Yoshiki Yomogida, Ali IzadiNajafabadi, Don N. Futaba and Kenji Hata. A stretchable carbon nanotube strain sensor for human-motion detection. Nat. Nanotechnol. 2011, 6, 296-301.

[9] Benjamin C-K. Tee, Chao Wang, Ranulfo Allen and Zhenan Bao. An electrically and mechanically self-healing composite with pressure- and flexion-sensitive properties for electronic skin applications. Nat. Nanotechnol. 2012, 7, 825-832.

[10] Daniel J. Cohen, Debkishore Mitra, Kevin Peterson and Michel M. Maharbiz. A Highly Elastic, Capacitive Strain Gauge Based on PercolatingNanotube Networks. Nano Lett. 2012, $12,1821-1825$.

[11] Darren J. Lipomi, Michael Vosgueritchian, Benjamin C-K. Tee, Sondra L. Hellstrom,Jennifer A. Lee, Courtney H. Fox and ZhenanBao. Skin-like pressure and strain sensors based on transparent elastic films of carbon nanotubes. Nat. Nanotechnol. 2011, 6, $788-792$.

[12] Zhong Lin Wang and Jinhui Song. Piezoelectric Nanogenerators Basedon Zinc Oxide Nanowire Arrays. Science, 2006, 312, 242-245. 
[13] Xu Xiao, Longyan Yuan, Junwen Zhong, Tianpeng Ding, Yu Liu, Zhixiang Cai, Yaoguang Rong, Hongwei Han, Jun Zhou and Zhong Lin Wang. High-Strain Sensors Based on ZnO Nanowire/Polystyrene Hybridized Flexible Films. Adv. Mater. 2011, 23, 5440-5444. [14] Jin Yang, Jun Chen, Yuanjie Su, Qingshen Jing, Zhaoling Li, Fang Yi, Xiaonan Wen,Zhaona Wang and Zhong Lin Wang. Eardrum-Inspired Active Sensors for Self-Powered Cardiovascular System Characterization and Throat-Attached Anti-Interference Voice Recognition. 2015, 27, 1316-1326.

[15] E. Fukada. PIEZOELECTRICITY OF BIOPOLYMERS. Biorheology, 1995, 32, 593609.

[16] J. Kobayashi, T. Asahi, M. Ichiki, A. Oikawa, H. Suzuki. Structural and optical properties of poly lactic acids. J. Appl. Phys. 1995, 77, 2957-2973.

[17] Y. Ikada, Y. Shikinami Y. Hara, M. Tagawa and E. Fukada. Enhancement of bone formation by drawn poly(L-lactide). J. Biomed. Mater. Res., 1996, 30, 553-558.

[18] Eiichi Fukada. History and Recent Progress in Piezoelectric Polymers. IEEE Trans. Ultrason., Ferroelect., Freq. Contro. 2000, 47, 1277-1290.

[19] Eiichi Fukada. New Piezoelectric Polymers. Jpn. J. Appl. Phys., 1998, 37, 2775-2780.

[20] Masamichi Ando, Hideki Kawamura, Hiroaki Kitada, Yasuyuki Sekimoto, Takafumi Inoue, and Yoshiro Tajitsu. Pressure-Sensitive Touch Panel Based on Piezoelectric Poly(Llactic acid) Film. Jpn. J. Appl. Phys., 2013, 52, 09KD17 (1-4).

[21] Masamichi Ando, Hideki Kawamura, Keisuke Kageyama, and Yoshiro Tajitsu. Film Sensor Device Fabricated by a Piezoelectric Poly(L-lactic acid) Film. Jpn. J. Appl. Phys., 2012, 51, 09LD14 (1-4).

[22] Sujoy Kumar Ghosh, Dipankar Mandal. Efficient natural piezoelectric nanogenerator: Electricity generation from fish swim bladder .Nano Energy 28 (2016) 356-365. 
[23] Sujoy Kumar Ghosh and Dipankar Mandal. Bio-assembled, piezoelectric prawn shell made self-powered wearable sensor for non-invasive physiological signal monitoring. APPLIED PHYSICS LETTERS 110, 123701(1-5) (2017).

[24] Md. Mehebub Alam and Dipankar Mandal. Native Cellulose Microfiber-Based Hybrid Piezoelectric Generator for Mechanical Energy Harvesting Utility. ACS Appl. Mater. Interfaces 2016, 8, 1555-1558.

[25] Sujoy Kumar Ghosh, Prakriti Adhikary, Santanu Jana, Anirban Biswas, Vitor Sencadas, Sudipto Dutta Gupta, Bipan Tudu, Dipankar Mandal. Electrospun gelatin nanofiber based self-powered bio-e-skin for health care Monitoring. Nano Energy 36 (2017) 166-175.

[26] Byung Yang Lee, Jinxing Zhang, Chris Zueger, Woo-Jae Chung, So Young Yoo, Eddie Wang, Joel Meyer, Ramamoorthy Ramesh and Seung-Wuk Lee. Virus-based piezoelectric energy generation. Nat. Nanotechnol. 7 (2012) 351-356..

[27] Qing Yang Pan, Tasaka Shigeru and Inagaki Norihiro. Ferroelectric behavior in poly-Llactic acid.Jpn. J. Appl. Phys., 1996, 35, L1442-L1445.

[28] Sol Jee Lee, ArunAnandPrabu, Kap Jin Kim. Piezoelectric propertiesofelectrospunpoly(L-lactic acid) nanofiber web. Mater Lett.,2015, 148, 58-62.

[29] T. A. M. Valente, D. M. Silva, P. S. Gomes, M. H. Fernandes, J. D. Santos, and V. Sencadas. Effect of Sterilization Methods on Electrospun Poly(lactic acid) (PLA) Fiber Alignment for Biomedical Applications. ACS Appl. Mater. Interfaces. 2016, 8, 3241-3249.

[30] Clarisse Ribeiro, Vitor Sencadas, Carlos Miguel Costa,José Luís Gómez Ribelles and Senentxu Lanceros-Méndez. Tailoring the morphology and crystallinity of poly(L-lactide acid) electrospun membranes. Sci. Technol. Adv. Mater., 2011,12, 015001 (1-9).

[31] Nan Jing, Xiaoting Jiang, Qian Wang, Yongjiao Tang and Pudun Zhang. Attenuated total reflectance/Fourier transforminfrared (ATR/FTIR) mapping coupled with principal component analysis for the study of in vitro degradation of porous polylactide/hydroxyapatite 
composite material. Anal. Methods, 2014, 6, 5590-5595.

[32] Subrata Maji, Piyush Kanti Sarkar, Leena Aggarwal, Sujoy Kumar Ghosh, Dipankar Mandal, Goutam Sheet and Somobrata Acharya. Self-oriented b-crystalline phase in the polyvinylidene fluoride ferroelectric and piezo-sensitive ultrathin Langmuir-Schaefer film. Phys. Chem. Chem. Phys.,2015, 17, 8159-8165.

[33] Yangjiang Wu, Qingzhao Gu, Guangzhu Ding, Fuqiang Tong, Zhijun Hu and Alain M. Jonas. Confinement Induced Preferential Orientation of Crystals and Enhancement of Properties in Ferroelectric Polymer Nanowires. ACS Macro Lett., 2013, 2, 535-538.

[34] Long Gu, Nuanyang Cui, Li Cheng, Qi Xu, SuoBai, Miaomiao Yuan, Weiwei Wu, Jinmei Liu,Yong Zhao, Fei Ma, Yong Qin and Zhong Lin Wang.Flexible Fiber Nanogenerator with 209 V Output Voltage Directly Powers a Light-Emitting Diode. Nano Lett., 2013, 13, 91-94.

[35] Xiao-Sheng Zhang, Meng-Di Han, Ren-Xin Wang, Fu-Yun Zhu, Zhi-Hong Li, Wei Wang and Hai-Xia Zhang. Frequency- Multiplication High -Output Triboelectric Nanogenerator for Sustainably Powering Biomedical Microsystems. Nano Lett. 2013, 13, $1168-1172$.

[36] Ayesha Sultana, Md. MehebubAlam, Anirban Biswas, Tapas Ranjan Middya and Dipankar Mandal. Fabrication of wearable semiconducting piezoelectric nanogenerator made with electrospun-derived zinc sulphide nanorods and poly(vinyl alcohol) nanofibers. Transl. Mater. Res., 2016, 3, 045001 (1-11).

[37] Ayesha Sultana, Md. Mehebub Alam,Samiran Garain, Tridib Kumar Sinha, Tapas Ranjan Middya and Dipankar Mandal. An Effective Electrical Throughput from PANI Supplement ZnS Nanorods and PDMS-Based Flexible Piezoelectric Nanogenerator for Power up Portable Electronic Devices: An Alternative of MWCNT Filler. ACS Appl. Mater. Interfaces., 2015, 7, 19091-19097. 
[38] Luana Persano, Canan Dagdeviren , Claudio Maruccio , Laura De Lorenzis , and Dario Pisignano. Cooperativity in the Enhanced Piezoelectric Response of Polymer Nanowires. Adv. Mater. 2014, 26, 7574-7580.

[39] Sujoy Kumar Ghosh, Tridib Kumar Sinha, Biswajit Mahanty, Santanu Jana and Dipankar Mandal. Porous polymer composite membrane based nanogenerator: A realization of self-powered wireless green energy source for smart electronics applications. J. Appl. Phys., 2016, 120, 174501 (1-11).

[40] Conor S. Boland, Umar Khan, Claudia Backes, Arlene O’Neill, Joe McCauley, Shane Duane, Ravi Shanker, Yang Liu, Izabela Jurewicz, Alan B. Dalton and Jonathan N. Coleman. Sensitive, High-Strain, High-Rate Bodily Motion Sensors Based on Graphene_Rubber Composites. ACS Nano, 2014, 8, 8819-8830.

[41] Morteza Amjadi, Yong Jin Yoon and Inkyu Park. Ultra-stretchable and skin-mountable strain sensors using carbon nanotubes-Ecoflex nanocomposites. Nanotechnology, 2015, 26, $375501(1-11)$.

[42] Cátia Leitão, Lúcia Bilro, Nélia Alberto, Paulo Antunes, Hugo Lima, Paulo S. André, RogérioNogueira, and João L. Pinto. Feasibility studies of Bragg probe for noninvasive carotid pulse waveform assessment. J. Biomed. Opt., 2013, 18, 017006-017006.

[43] Yasser Khan,Aminy E. Ostfeld, Claire M. Lochner, Adrien Pierre, and Ana C. Arias.Monitoring of Vital Signs with Flexible and WearableMedical Devices. Adv. Mater. 2016, 28, 4373-4395.

[44] Alfredo L. Pauca, Michael F. O'Rourke, Neal D. Kon. Prospective Evaluation of a Method for EstimatingAscending Aortic Pressure From the Radial Artery Pressure Waveform. Hypertension 2001, 38, 932-937. 